\title{
Cardiac cell and gene therapies: two trajectories, one goal
}

\author{
Roger Hajjar and Valentin Fuster
}

Advances in our understanding of the molecular basis of ventricular dysfunction and the evolution of increasingly efficient gene transfer technology have placed congestive heart failure within reach of gene-based therapy. The road from basic mechanisms to clinical gene therapy for cardiac disease has been long and arduous, with intense animal and cellular experimentation that has often failed.

The application of cell-based therapies has followed a different trajectory. Only a few months elapsed between the first reports that showed beneficial effects of bone-marrowderived stem cells after myocardial infarction in rodents and the initiation of clinical trials. Since then, a large number of trials have been conducted using stem cells derived from a variety of sources, the success of which has been quite modest. As we pointed out in our editorial in January 2007 (Nadal-Ginard and Fuster [2007] Nat Clin Pract Cardiovasc Med 4: 1) the challenges for cell therapy remain at the basic mechanistic level: identifying suitable cells for effective cardiomyocyte regeneration, recreating the appropriate microenvironment for enabling progenitor cells to survive and differentiate, and developing safe and effective methods of delivery.

Although meta-analyses of the randomized controlled trials have demonstrated that intracoronary infusion and ventricular administration of bone-marrow-derived stem cells is safe in patients with ischemic heart disease and is associated with very slight improvement of the left-ventricular ejection fraction, this improvement only lasts for 3-6 months. Additionally, these cells do not differentiate into mature cardiomyocytes. Skeletal-myoblast-based therapy has also been disappointing; delivery of these cells is associated with arrhythmias and no improvement in function, or, if beneficial effects of delivering these cells are detected, they disappear within 6-12 months.
The road

from basic

mechanisms

to clinical

gene therapy

for cardiac

disease has

been long and

arduous...

$R$ Hajjar is the Arthur and Janet $C$ Ross Professor of Medicine and Director of the

Cardiovascular

Research Center,

Department of

Cardiovascular

Medicine, Mount Sinai

School of Medicine,

New York, NY, USA.

$V$ Fuster is the Editorin-Chief of Nature

Clinical Practice

Cardiovascular

Medicine.

Competing interests
R Hajjar has declared
associations with the
following companies:
Celladon and Nanocor.
Please see the article
online for details of the
relationships. V Fuster has
declared no competing
interests.
www.nature.com/clinicalpractice
doi:10.1038/ncpcardio1411

\section{Competing interests}

$\mathrm{R}$ Hajjar has declared associations with the

Celladon and Nanocor.

online for details of the relationships. V Fuster has declared no competing

www. nature.com/clinicalpractice doi:10.1038/ncpcardio1411
Increasing evidence points towards the presence of resident cardiac stem cells with multipotent progenitor potential. These putative cardiac stem cells are clonogenic and have the capacity to self-renew and differentiate into cardiomyocytes, smooth muscle cells and endothelial cells. The potential advantages of autologous cardiac stem cells are their cardiomyogenic potential and their purported ability to be targeted by small molecules and external factors. There is a surge to deliver these cells to patients as soon as possible despite major hurdles-including expansion in culture, phenotyping, genotyping, engraftment and survivalnot being resolved.

In the last year or so, there has been a major breakthrough in the field of stem cell biology: researchers have demonstrated that normal human skin cells can be reprogrammed to an embryonic state, producing induced pluripotent stem cells. This finding potentially provides an alternative method of producing patientspecific cells that can differentiate into cardiomyocytes. Already, the urge to quickly translate this exciting finding to the clinical setting has been mounting, even though the potential for these induced pluripotent stem cells to grow uncontrollably is still largely unknown.

The challenges facing stem cell therapy continue to grow with the explosion of discoveries in this field; however, these challenges have not stemmed the tide of clinical trials being started. On the other hand, after a decade of painstaking experimentation, the first clinical trial (Phase 1) of gene therapy in patients with congestive heart failure (Hajjar RJ et al. [2008] $J$ Card Fail 14: 355-367) has been completed by our group. Cell and gene therapies for patients with heart failure are taking quite different trajectories. Although it is hard to pre-judge the final outcome, we remain aware of the lessons learned from Aesop's fable about the hare and the tortoise- "slow and steady wins the race". 